Supporting Information for

\title{
Electrolyte Regulation of Bismuth Ions toward High- Performance Aqueous Manganese-based Batteries
}

\author{
Mingming Wang ${ }^{\dagger}$, Yahan Meng ${ }^{\dagger}$, Na Chen, Mingyan Chuai, Chunyue Shen, Xinhua \\ Zheng, Yuan Yuan, Jifei Sun, Yan Xu, Wei Chen* \\ Department of Applied Chemistry, School of Chemistry and Materials Science, Hefei \\ National Laboratory for Physical Sciences at the Microscale, University of Science and \\ Technology of China, Hefei, Anhui 230026, China
}

*Corresponding author: weichen1@ustc.edu.cn

${ }^{7}$ These authors contributed equally to this work. 


\section{Experimental:}

\section{Materials}

The chemicals and materials in this work are all commercially available and used as received: chromium (III) chloride hexahydrate $\left(\mathrm{CrCl}_{3} \cdot 6 \mathrm{H}_{2} \mathrm{O}\right.$, Sinopharm Chemical Reagent Co., Ltd), manganese chloride tetrahydrate $\left(\mathrm{MnCl}_{2} \cdot 4 \mathrm{H}_{2} \mathrm{O}\right.$, Sigma Aldrich), bisumth chloride $\left(\mathrm{BiCl}_{3}\right.$, Sinopharm Chemical Reagent Co., Ltd), sulfuric acid $\left(\mathrm{H}_{2} \mathrm{SO}_{4}\right.$, Sinopharm Chemical Reagent Co., Ltd), anion exchange membrane (FAP-450, Fuel Cell Store, USA), carbon felt (Dalian Longtian Tech. Co.), Pt foil (Sigma Aldrich, 99.9\%), graphite rod (Alfa Aesar, 99.9995\%) and deionized water (resistance of 18.2 $\mathrm{M} \Omega$, Milli Q)

\section{Characterizations}

Scanning electron microscope (SEM, Hitachi 8220, Japan) was carried out to characterize the morphologies and structures of the electrodes. X-ray photoelectron spectroscopy (Thermo ESCALAB 250Xi) was performed with Al $\mathrm{K}_{\alpha}$ source. X-ray diffraction (XRD) patterns were collected on a Philips X'Pert PRO SUPER X-ray diffractometer equipped with graphite monochromatized $\mathrm{Cu} \mathrm{K}_{\alpha}$ radiation.

\section{Electrochemical tests}

The battery cycling performance was tested on battery test systems (LandHe, Wuhan, China and Neware, Shenzhen, China). The galvanostatic charge-discharge rate capability, cyclic voltammetry, and the electrochemical impedance spectra of the three- 
electrode systems as well as the two-electrode full cells were carried out on a VMP3 electrochemical workstation (Bio-Logic, France). The EIS was tested in a frequency range of $100 \mathrm{kHz}$ to $1 \mathrm{~Hz}$. In the three-electrode measurements, carbon felt, graphite rod and $\mathrm{Ag} / \mathrm{AgCl}$ were used as the working, counter and reference electrodes, respectively. In the fabrication of the two-electrode full cell, two carbon felts were acting as the cathode and anode current collectors. In the three-electrode system and the full battery, the electrolyte consists of $1 \mathrm{M} \mathrm{CrCl}_{3}, 1 \mathrm{M} \mathrm{MnCl}_{2}, 0.5 \mathrm{M} \mathrm{H}_{2} \mathrm{SO}_{4}$ with or without $0.001 \mathrm{M} \mathrm{BiCl}_{3}$ in water. All electrochemical tests were conducted at room temperature $\left(\sim 25^{\circ} \mathrm{C}\right)$. 


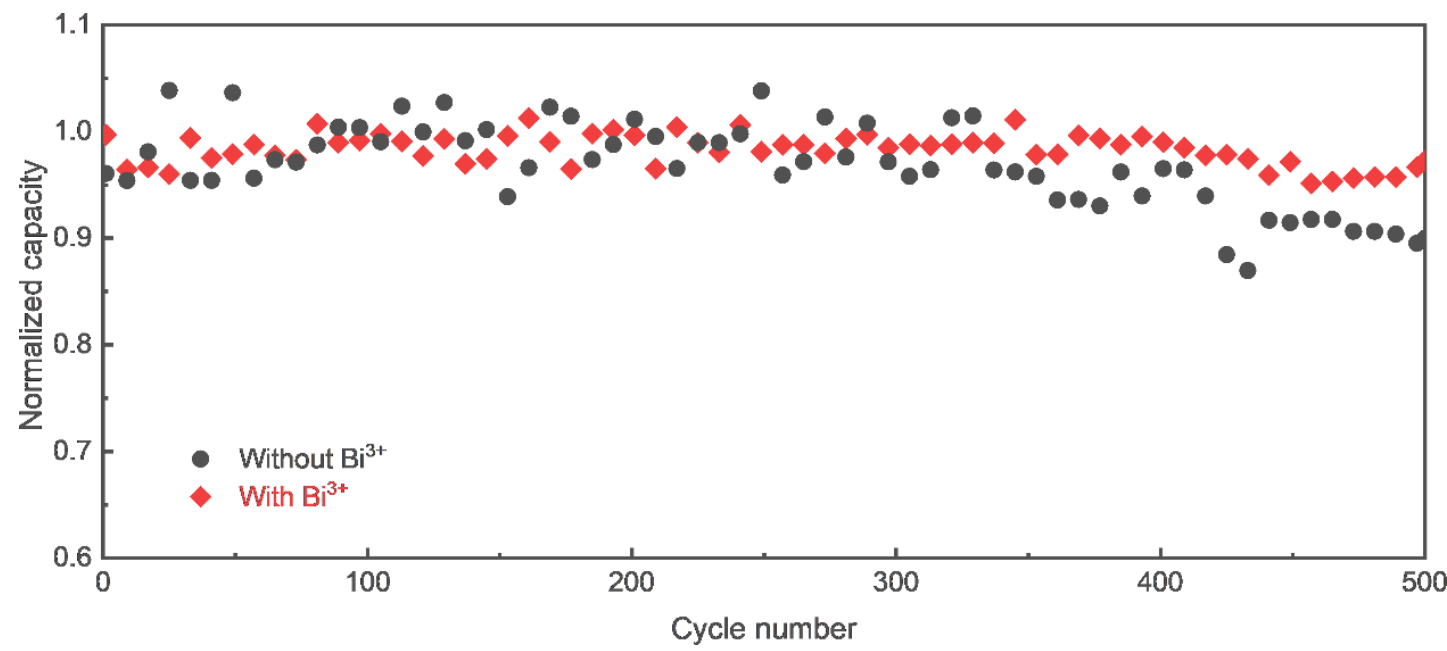

Supplementary Figure 1. Long-term cycling performance of the $\mathrm{MnO}_{2}$ cathode with and without $\mathrm{Bi}^{3+}$ in the electrolytes at a current density of $100 \mathrm{~mA} \mathrm{~cm}^{-2}$ for 500 cycles. 

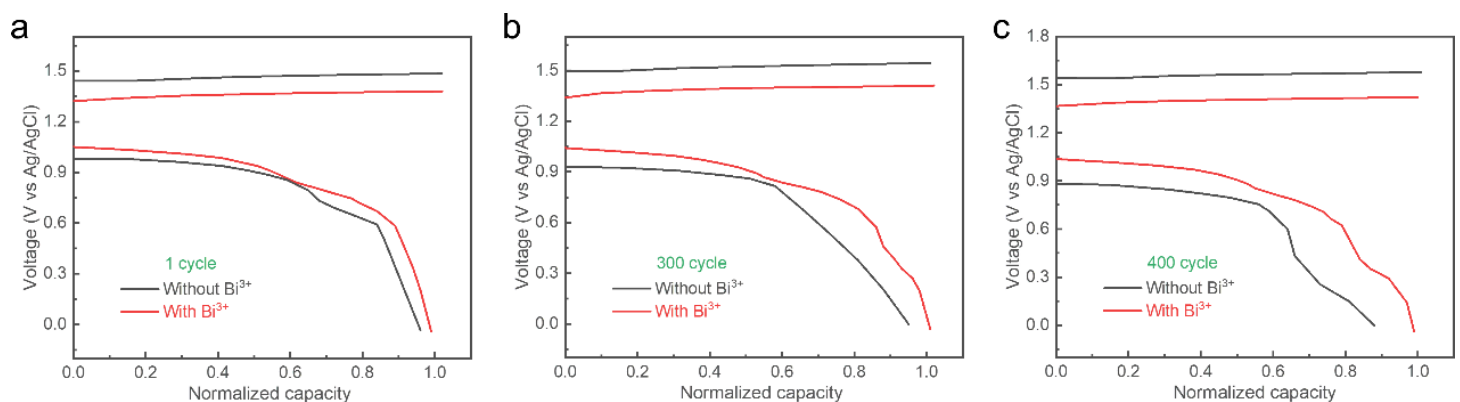

Supplementary Figure 2. The GCD curves of the $\mathrm{MnO}_{2}$ cathode with and without $\mathrm{Bi}^{3+}$ in the electrolytes in different cycles at a current density of $100 \mathrm{~mA} \mathrm{~cm}^{-2}$. 

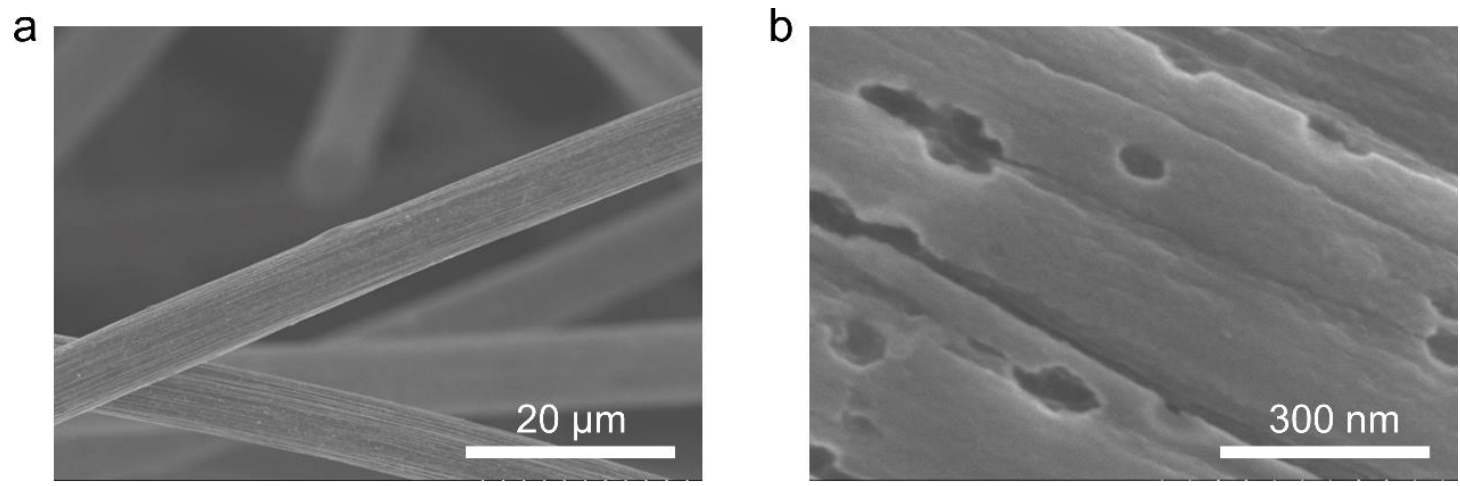

C

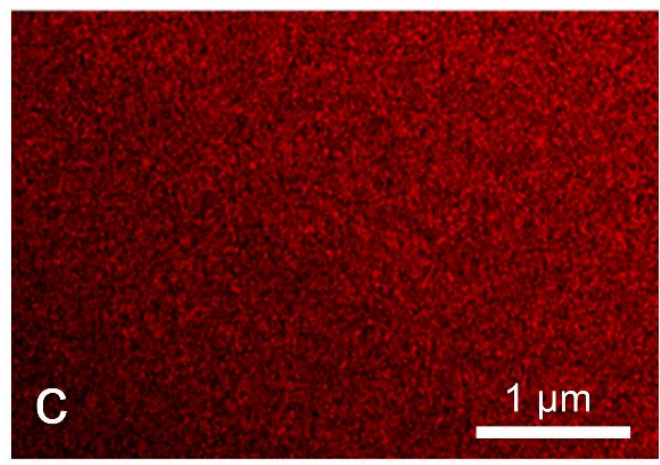

d

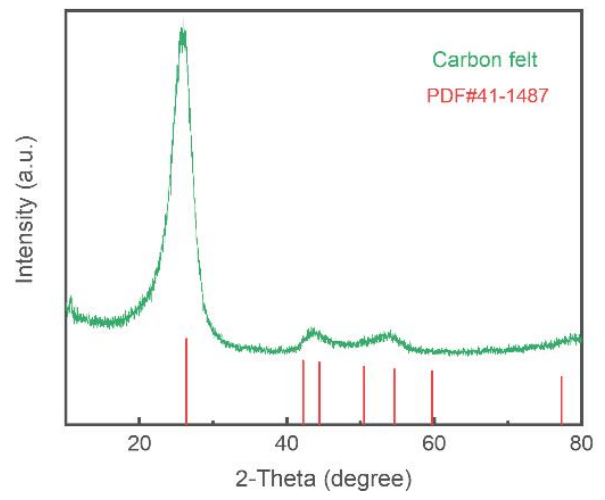

Supplementary Figure 3. SEM (a-b), elemental mapping (c) and XRD (d) of the pristine carbon felt. 
a

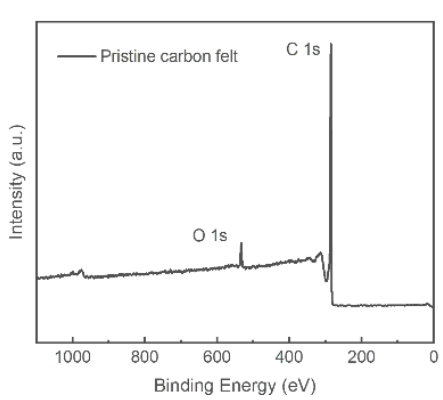

b

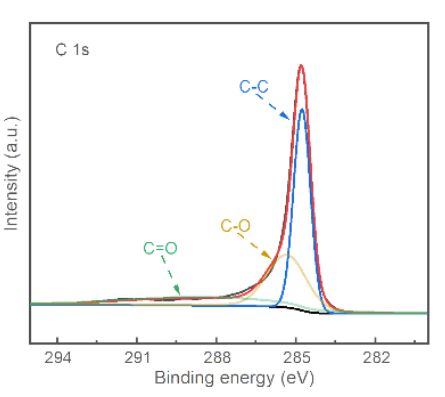

C

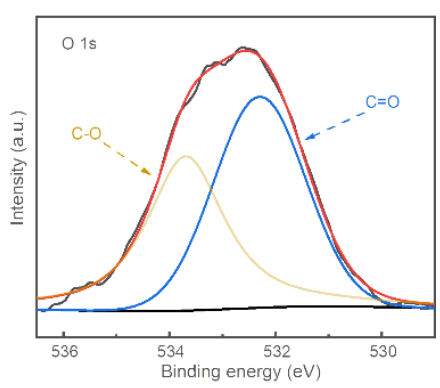

Supplementary Figure 4. (a) XPS survey spectra of the pristine carbon felt. Highresolution XPS spectra of carbon felt for C 1s (b) and O 1s (c). 

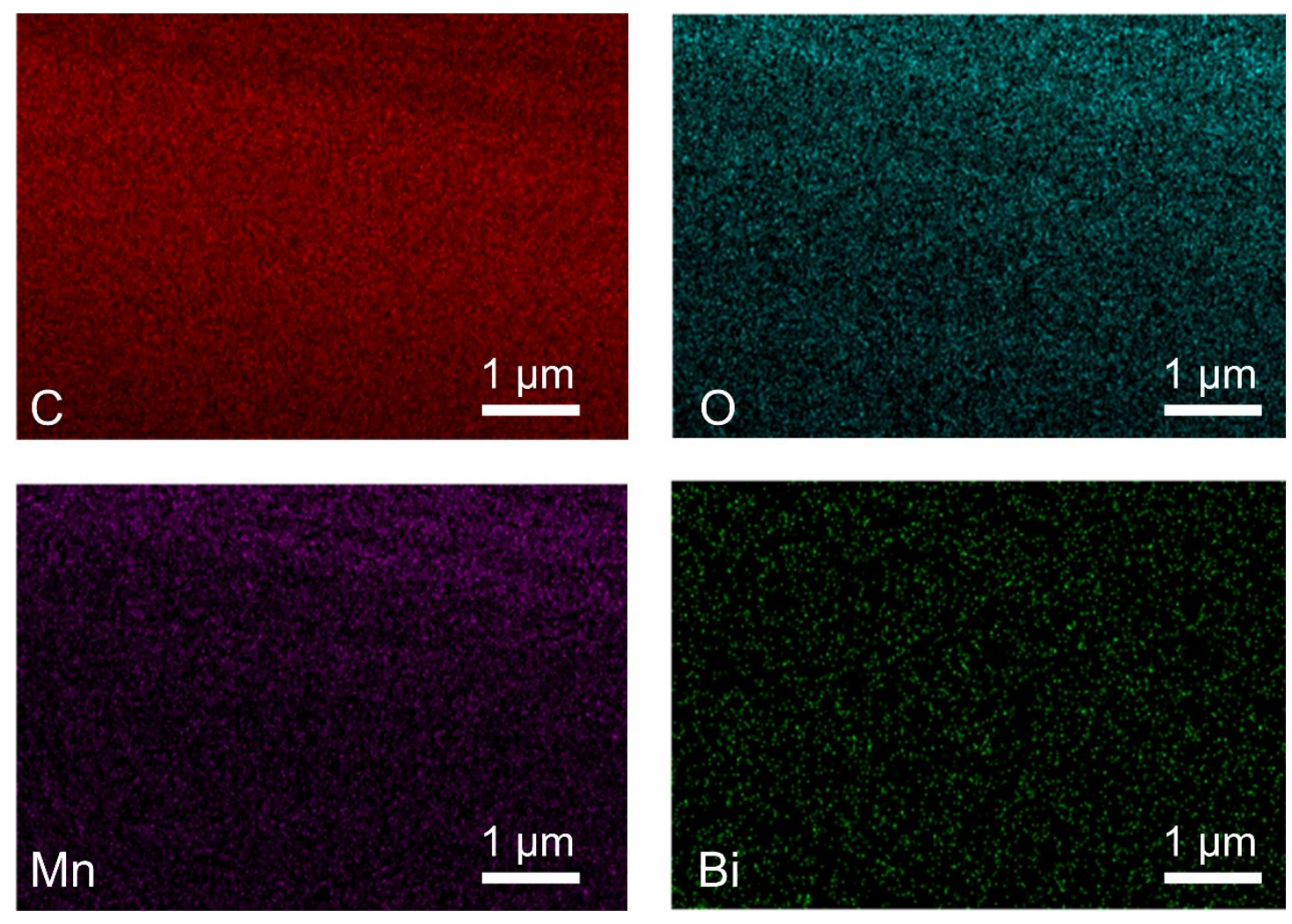

Supplementary Figure 5. EDX elemental mapping of C, Mn, O and Bi in SEM mode for the cathode $\mathrm{Bi}-\mathrm{MnO}_{2}$ coated carbon felt. 


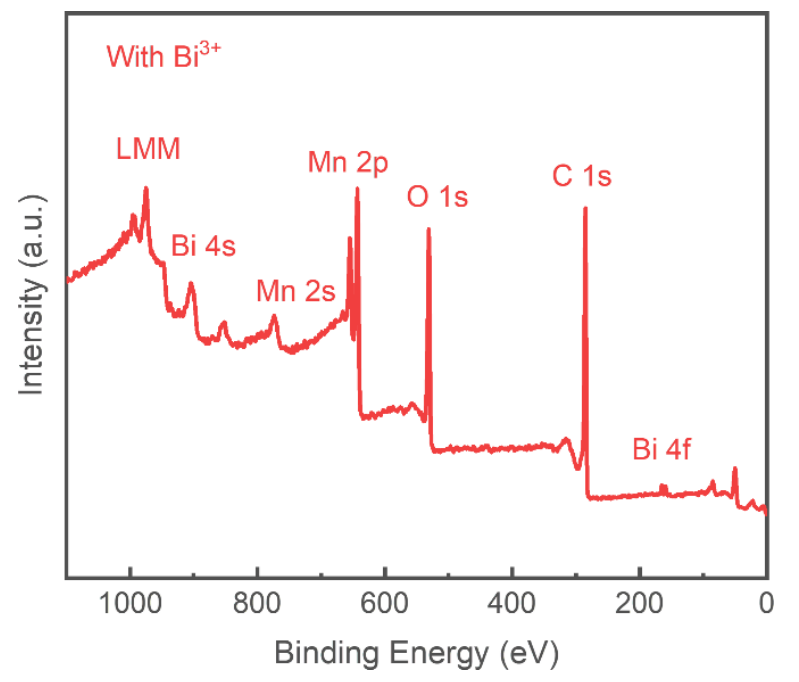

Supplementary Figure 6. XPS survey spectra of carbon felt in cathode with $\mathrm{Bi}^{3+}$ in the electrolytes. 


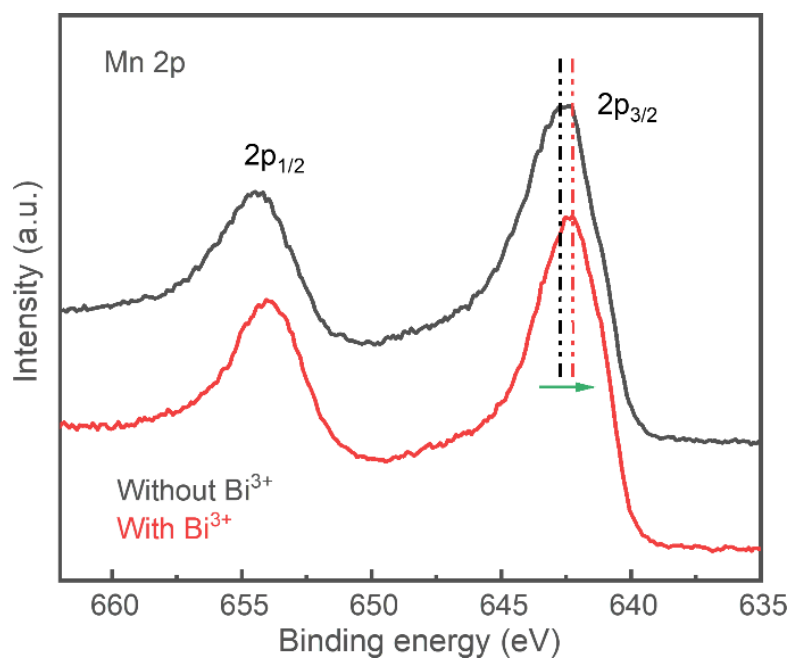

Supplementary Figure 7. The high-resolution XPS spectra of Mn $2 p$ for the cathode $\mathrm{MnO}_{2}$ without and with $\mathrm{Bi}^{3+}$ in the electrolytes. 

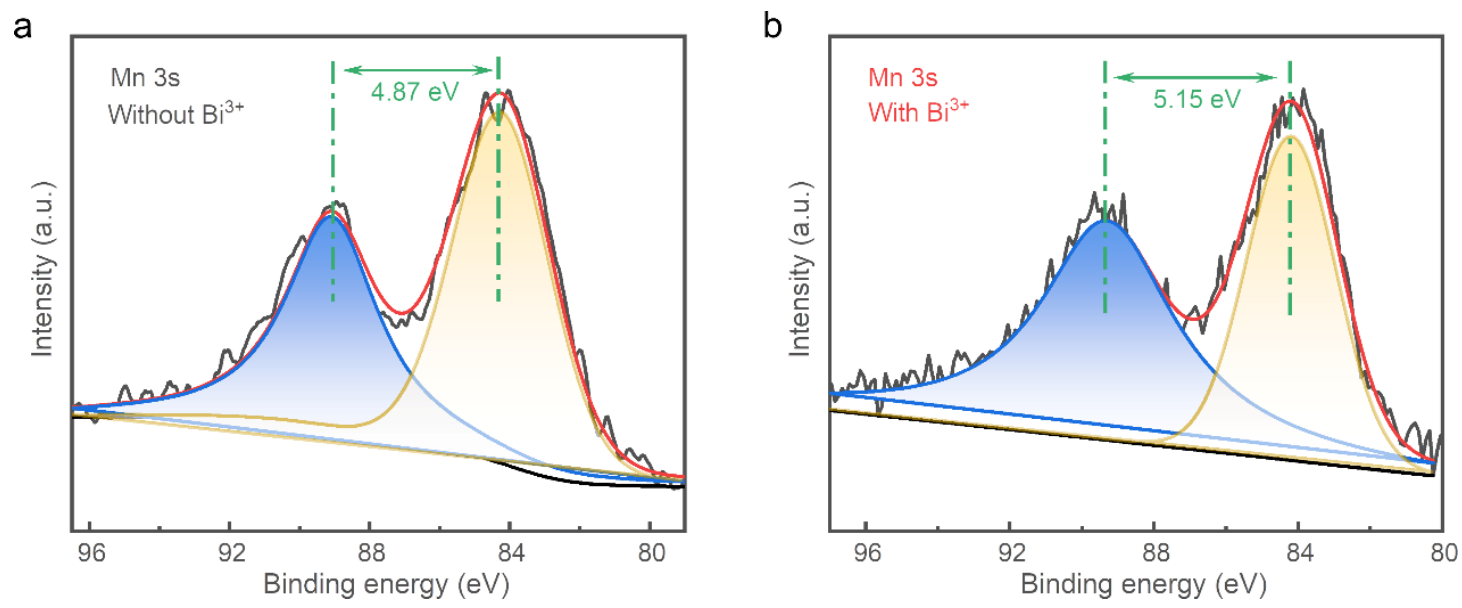

Supplementary Figure 8. (a) and (b) are high-resolution XPS spectra of Mn 3s for the cathode $\mathrm{MnO}_{2}$ without and with $\mathrm{Bi}^{3+}$ in the electrolytes. The value of $4.87 \mathrm{eV}$ and $5.15 \mathrm{eV}$ refer to the $\mathrm{Mn} 3 \mathrm{~s}$ peak splitting energy of the $\mathrm{MnO}_{2}$ on the cathode. 


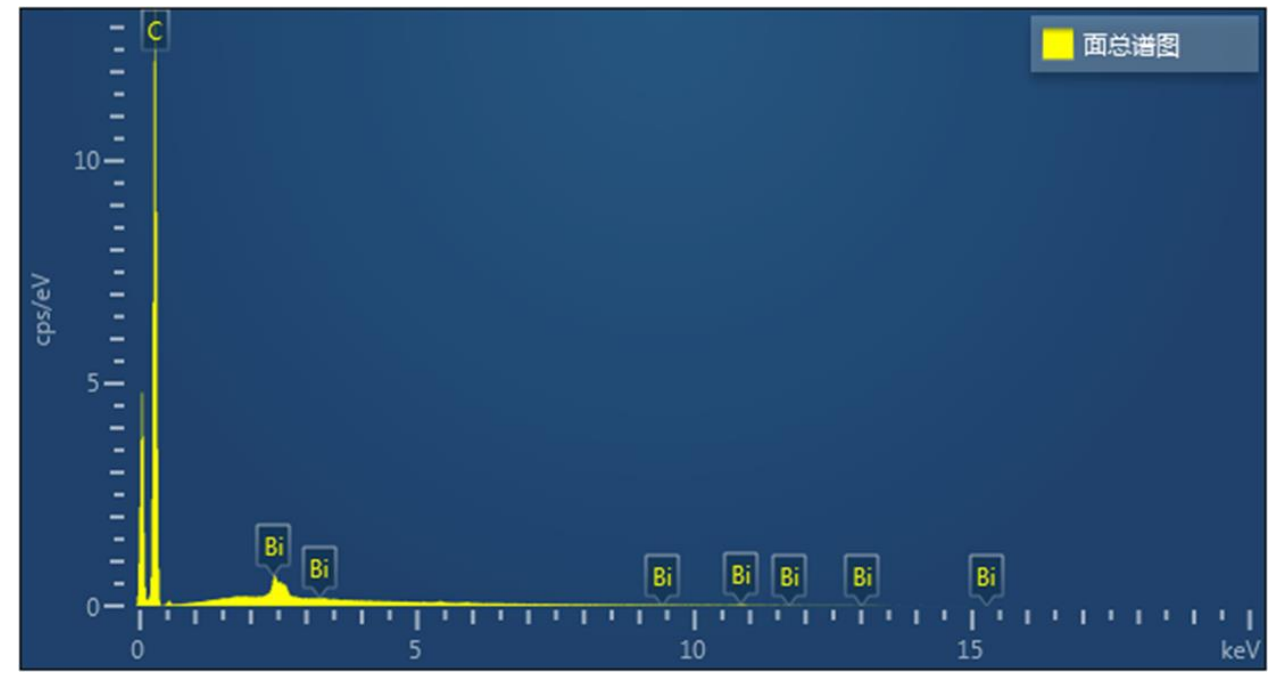

Supplementary Figure 9. EDS mapping of metallic Bi nanoparticles on the anode electrode surface. 


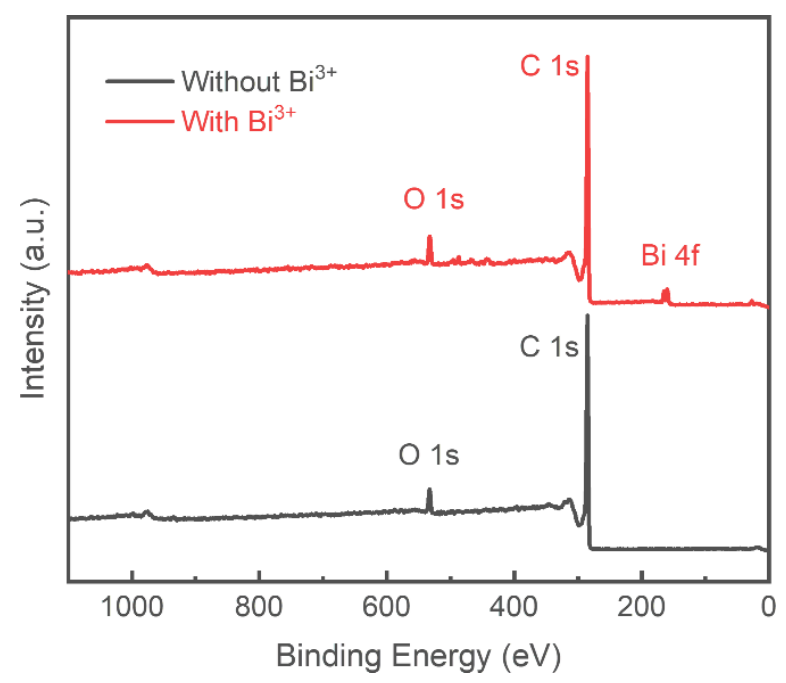

Supplementary Figure 10. XPS survey spectra of the anode carbon felt with and without $\mathrm{Bi}^{3+}$ in the electrolytes. 

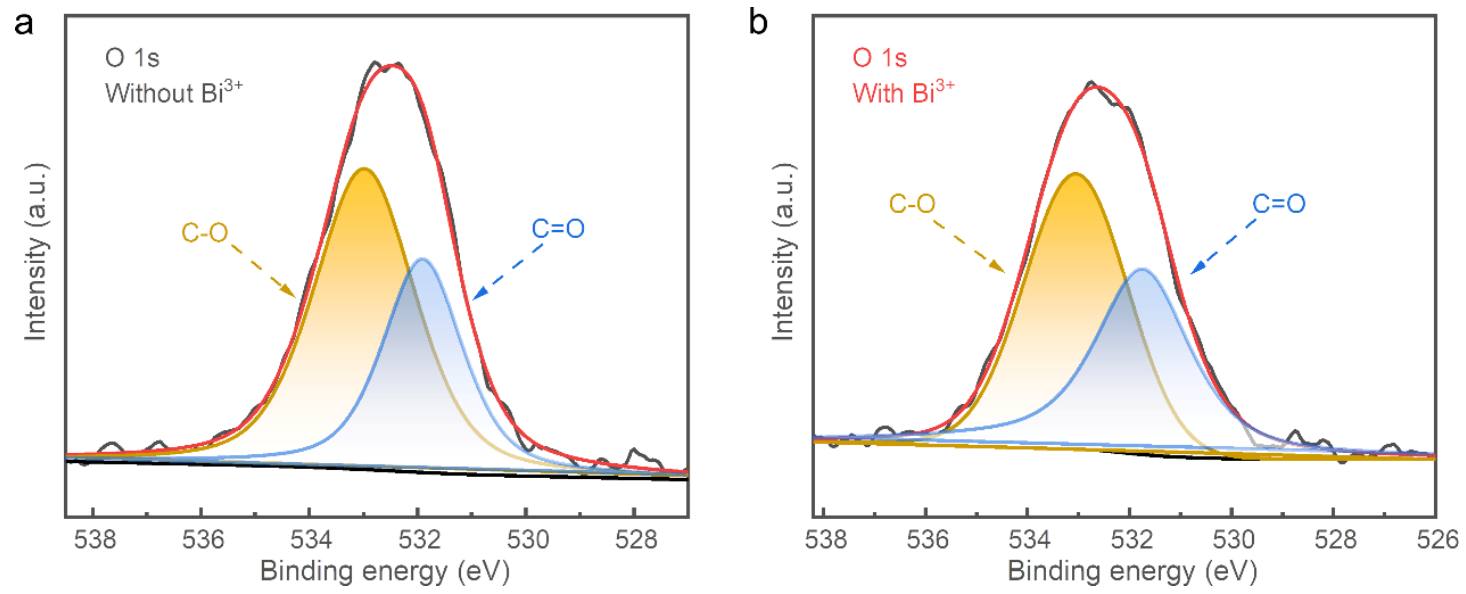

Supplementary Figure 11. High-resolution XPS spectra of O 1s for the anode carbon felt without $\mathrm{Bi}^{3+}(\mathbf{a})$ and with $\mathrm{Bi}^{3+}(\mathbf{b})$. 


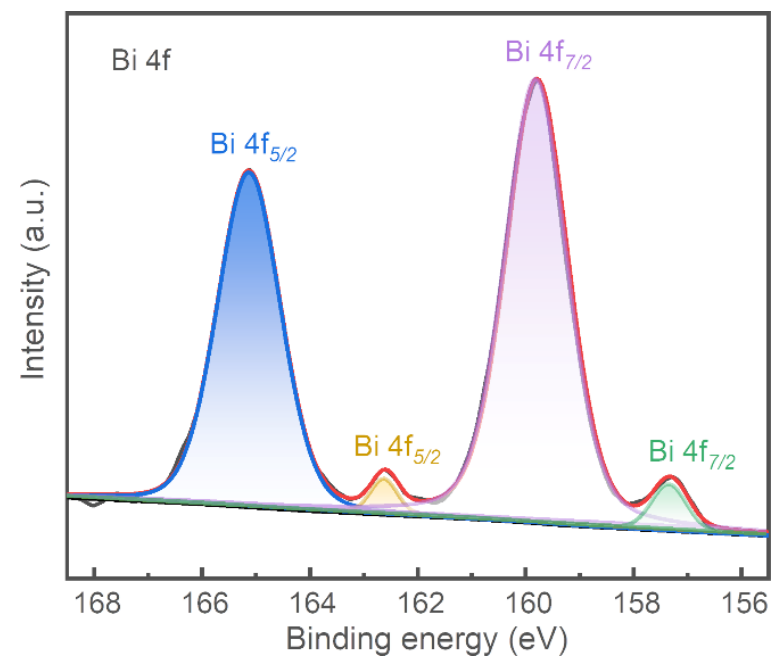

Supplementary Figure 12. High-resolution XPS spectra of Bi $4 \mathrm{f}$ for the anode carbon felt after charge in the $\mathrm{Bi}^{3+}$ regulated electrolyte. 

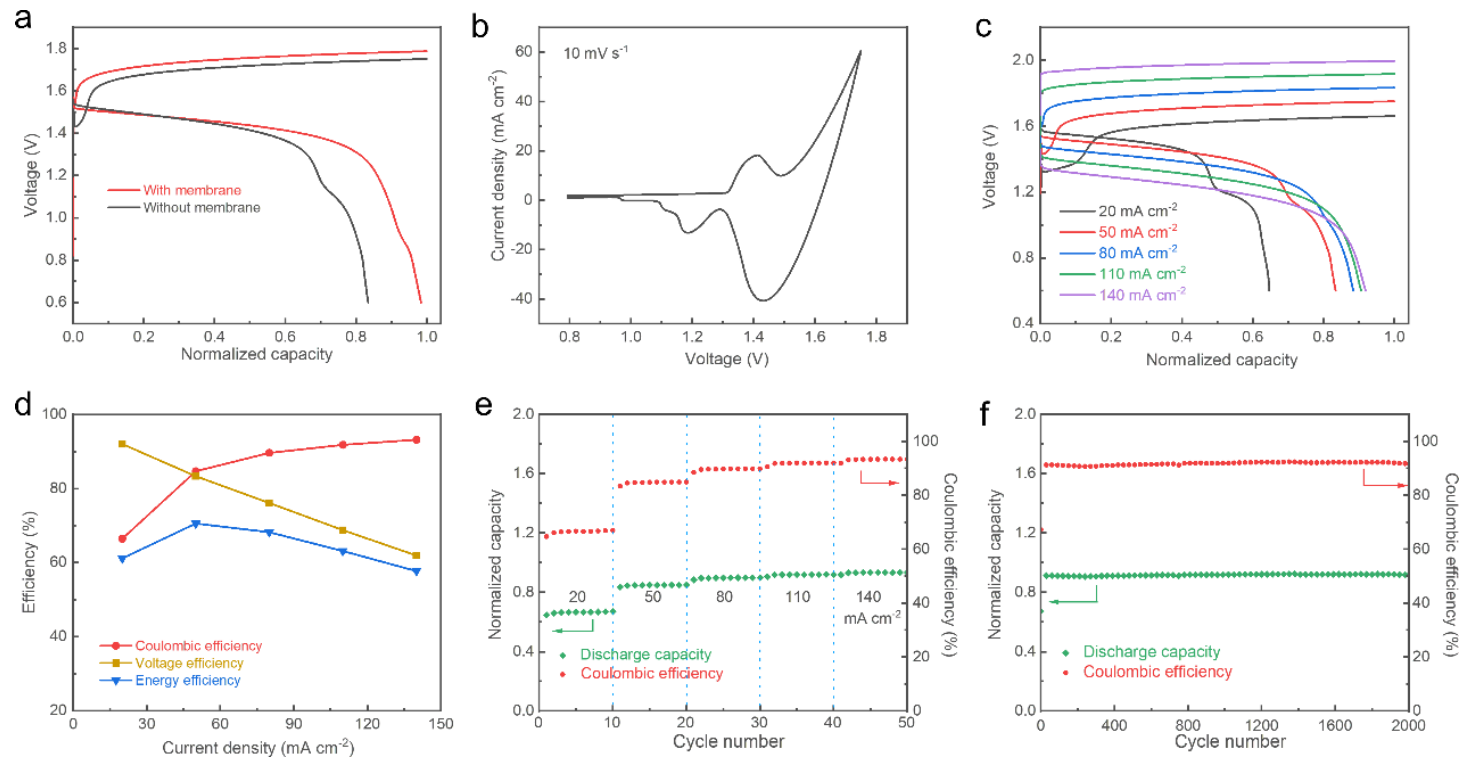

Supplementary Figure 13. Electrochemical performance of the static $\mathrm{MnO}_{2}-\mathrm{Cr}(\mathrm{Bi})$ battery without AEM. (a) Charge/discharge profiles at a current density of $50 \mathrm{~mA} \mathrm{~cm}^{-2}$ with and without using FAP-450 anion exchange membrane as the separator. (b) Cyclic voltammetry curves at a scan rate of $10 \mathrm{mV} \mathrm{s}^{-1}$. (c) Galvanostatic charge and discharge curves under different current densities from $20 \mathrm{~mA} \mathrm{~cm}^{-2}$ to $140 \mathrm{~mA} \mathrm{~cm}{ }^{-2}$. (d) Rate capability performance under different current densities. (e) Coulombic, voltage and energy efficiencies as a function of current density. (f) 2000 cycles of discharge capacity and Coulombic efficiency performance at a charge/discharge current density of $100 \mathrm{~mA}$ $\mathrm{cm}^{-2}$. 

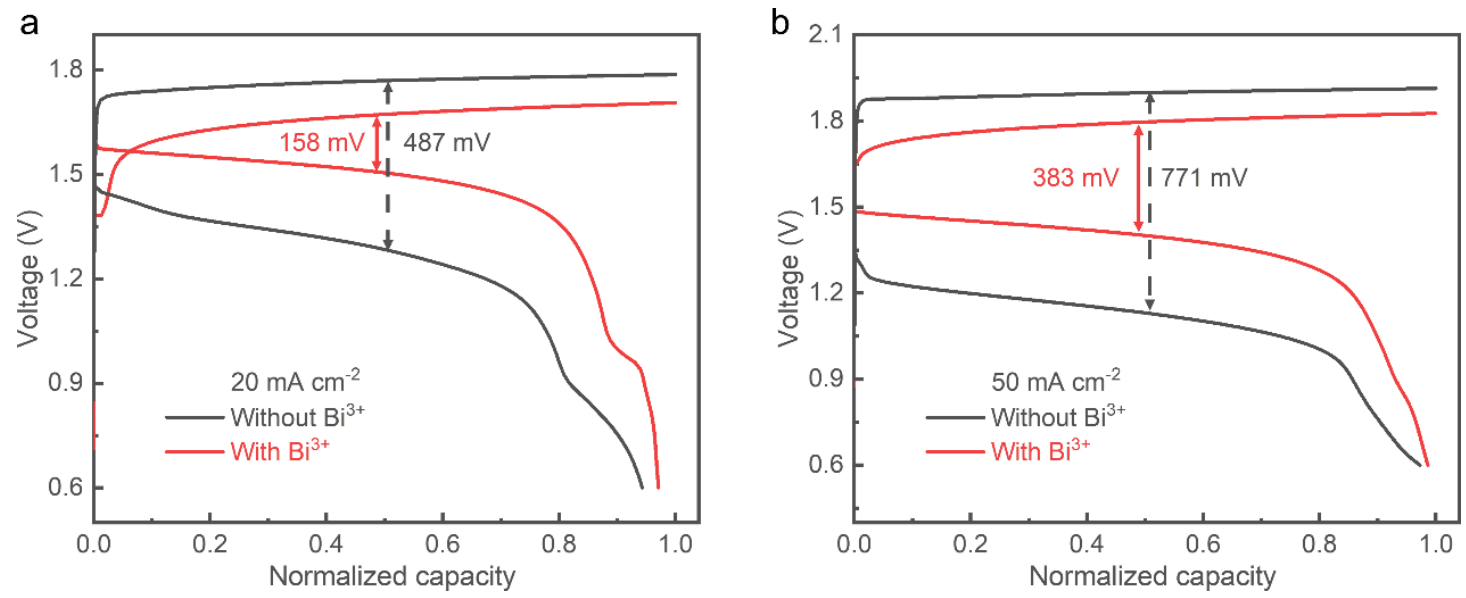

Supplementary Figure 14. The charge/discharge profiles at a current density of $20 \mathrm{~mA}$ $\mathrm{cm}^{-2}$ (a) and $50 \mathrm{~mA} \mathrm{~cm}^{-2}$ (b) for the static $\mathrm{MnO}_{2}-\mathrm{Cr}$ and $\mathrm{MnO}_{2}-\mathrm{Cr}(\mathrm{Bi})$ batteries. 


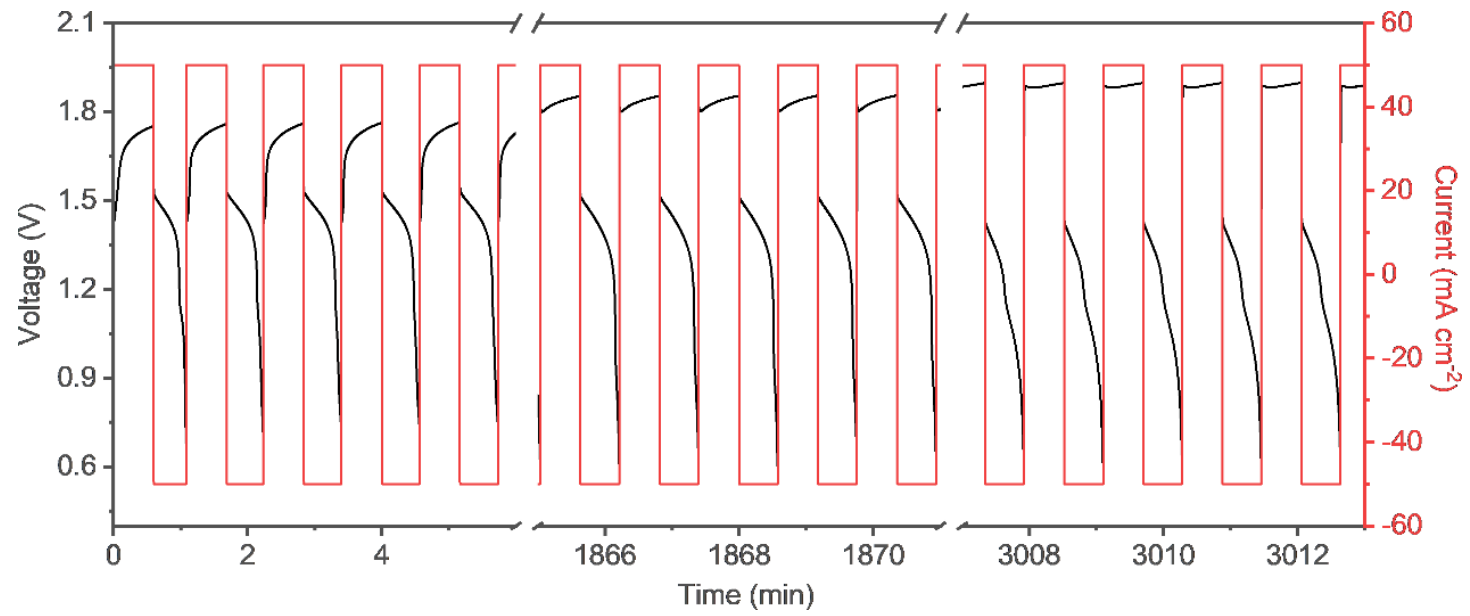

Supplementary Figure 15. The charge-discharge curves of the $\mathrm{MnO}_{2}-\mathrm{Cr}$ (Bi) static batteries at different cycles. 